\title{
Contribution of fine needle aspiration and auramine tests in diagnosis of Tuberculosis lymphadenitis at Kigali University Teaching Hospital (CHUK), Rwanda
}

\author{
Emile Musoni ${ }^{1}$, Alphonse Niyodusenga1*, Sylvain Iradukunda², Consolee Uwamahoro ${ }^{2}$ \\ ${ }^{1}$ University of Rwanda, College of Medicine and Health Sciences, School of Medicine and Pharmacy, \\ Department of Clinical Biology, Huye Campus, Rwanda \\ 2Institute of Applied Sciences, INES-Ruhengeri, Faculty of Applied Fundamental Sciences, Department of \\ Biomedical Laboratory Sciences, Musanze, Rwanda
}

*Corresponding author: Alphonse Niyodusenga, University of Rwanda, College of Medicine and Health Sciences, School of Medicine and Pharmacy, Department of Clinical Biology, Huye, Rwanda, E-mail:

niyodalph@yahoo.fr; Phone: (+250)788581592

\begin{abstract}
Background

Tuberculosis (TB) is an infection caused by Mycobacterium tuberculosis. Tuberculosis affects mainly the lungs but other organs of the body are involved. TB lymphadenitis is most common in extrapulmonary TB. Lymphadenitis is the infection or inflammation of the lymph nodes which are essential in immune response of the body.
\end{abstract}

\section{Objective}

To evaluate the contribution of fine needle aspiration (FNA) and auramine tests in the diagnosis of TB lymphadenitis.

\section{Methods}

Smears from lymph node aspirates were prepared. Air-dried smears were stained by auramine staining for AFB examination and Diff-Quick staining cytological technique to detect malignant cells and other pathology. The slides were examined by laboratory technologists and the pathologist.

\section{Results}

Results are based on a total number of 137 samples; 58 (42.33\%) cases were auramine positive for TB lymphadenitis while other 79 (57.67\%) suspected cases were non TB lymphadenitis. Necrotizing lymphadenitis, granulomatous lymphadenitis, chronic lymphadenitis, acute lymphadenitis and reactive lymphadenitis represented $21.19 \% ; 13.14 \% ; 1.42 \% ; 2.19 \%$; and $19.71 \%$ respectively.

\section{Conclusion}

The use of auramine test and FNA cytology should be considered as useful in the diagnosis of tuberculosis lymphadenitis. These techniques are less expensive, quick, safe and show low complication rate.

Keywords: TB lymphadenitis; fine needle aspiration; auramine test

\section{INTRODUCTION}

Tuberculosis is an airborne disease caused by the bacterium Mycobacterium tuberculosis. Mycobacterium tuberculosis is a non-motile, acidfast bacillus, obligate aerobe. The bacilli are 0.5-3 $\mu \mathrm{m}$ in length and have a very slow generation time of between 15 and 20 hours. The cell wall of the mycobacterium is unique in that it is composed mainly of acidic waxes, specifically mycolic acid, extraordinary lipid barrier and seven very closely related mycobacterial species $(M$. bovis, $M$. africanum, M. microti, $M$. laprae, $M$. pinnipedii, $M$. canetti and $M$. mungi). The causal agent of tuberculosis is Mycobacterium tuberculosis 
bacterium. The incubation period of tuberculosis is approximately 2 to 12 weeks.[1]

Inhalation is the most susceptible pathway of Mycobacterium tuberculosis infection. Mode of contamination of abdominal tuberculosis is by ingestion of infected food or milk or infected sputum.

Tuberculosis (TB) is a life-threatening disease which can affect any organ system, pulmonary tuberculosis being the predominant one. From the lungs $M$. tuberculosis can migrate through the blood or lymphatic pathways to other parts of the body, such as the kidney, spine, bones and brain. In case of extra-pulmonary tuberculosis, highly vascular areas such as lymph nodes, kidneys, spine, eyes, bones etc. are commonly affected.[2]

The abdominal TB, which is not common as pulmonary $\mathrm{TB}$, can be a cause of significant morbidity and mortality because it is usually diagnosed late due to its nonspecific clinical presentation.[3] From abdomen, rarely $M$. tuberulosis bacilli can enter into the portal circulation or into hepatic artery to affect internal organs like liver, pancreas and spleen.[4] The second pathway is hematogenous spread from tubercular focus from elsewhere in the body to abdominal organs such as kidneys, lymph nodes and peritoneum. The third pathway is direct spread to the peritoneum from infected adjacent foci, like the fallopian tubes, adnexa, or psoas muscle abscess. Finally it can spread through lymphatic pathways from nodes infected by $M$. tuberculosis.[5]

Worldwide, tuberculosis is a major health problem. According to the World Health Organization (WHO) report 2013, there were an estimated 8.6 million annual incidence of TB globally and 1.3 million people died from tuberculosis in 2012.[6]

Tuberculosis (TB) is a major cause of childhood morbidity and mortality in developing countries. In most cases, the pulmonary TB diagnosis is confirmed, if a sputum smear is positive. But more than $80 \%$ of children with TB have negative sputum smear, and extra-pulmonary TB is common in these patients. According to WHO, the evaluation of new techniques to improve the diagnosis and management of pediatric TB is an urgent research priority.[7]

Extra-pulmonary tuberculosis is defined according to WHO classification criteria as an infection by $M$. tuberculosis which affects tissues and organs outside the pulmonary parenchyma. It represents between 20 and $25 \%$ of all TB,[8] and lymphadenitis accounts for $17-43 \%$ of extrapulmonary cases.[9]

Tubercular lymphadenitis is the infection of lymph nodes caused by $M$. tuberculosis. Lymphadenitis occurs when the lymph node become enlarged by swelling or inflammation often in response to bacteria, viruses or fungi. Lymphadenitis spreads quickly because the lymph fluid passes through the bloodstream. Lymph nodes are essential for the initiation of immune responses by creating an environment in which lymphocytes and antigenpresenting cells can optimally interact.[10]

Tubercular lymphadenitis is the most common extra-pulmonary manifestation of tuberculosis. [11] In Hong Kong, from the Annual Report of Tuberculosis and Chest Service in 2004, TB lymphadenitis comprised $39.6 \%$ of all extrapulmonary tuberculosis. In Asians and Pacific islanders; lymph node tuberculosis constitutes 20$40 \%$ of extra-pulmonary tuberculosis; it is more common in children and women.[12]

Therefore, TB lymphadenitis should be considered among risk factors for TB-related death. Lymph vessels, like blood vessels, run all through the body. They carry lymph fluid and cells. Lymph nodes are small clumps of immune cells that act as filters for the lymphatic system. They also store white blood cells that help fight microorganisms. Once they are infected they lose that property. Although previously considered as a disease of childhood, TB lymphadenitis had a peak age of onset of 20 to 40 years, and in the United States it is most common in women and immigrants.[8]

The fine needle aspiration stained routinely by use of Diff-Quick staining may show the epithelioid cell granulomas, multinucleated giant cell; caseous necrosis and granulomas.[13] Fine needle aspiration (FNA) enables rapid, safe, relatively painless, and inexpensive sampling of lymph nodes. It does not require hospital admission or anaesthesia.[14] The Fine-needle aspiration cytology has become a popular tool in giving a rapid diagnosis within a matter of 20 minutes and is valuable as a screening procedure in patients with suspected mycobacterial infection, particularly in superficial, easily accessible locations such as cervical and submandibular lymph nodes. The 5 varied cytomorphologic appearances of tuberculosis have been reported on fine needle aspiration cytology such as granulomatous inflammation consisting of 
epithelioid, granulomas with or without Langhans giant cells, smears without granulomas showing predominantly necrotic material, occasional degenerated epithelioid cells with or without neutrophils.[15]

TB lymphadenitis is the most common extrapulmonary manifestation of tuberculosis. There are both diagnostic and therapeutic challenges because it mimics other pathologic processes and yields inconsistent physical and laboratory findings. Diagnosis is difficult often requiring biopsy. In this regard, the aim of this study was to evaluate the contribution of fine needle aspiration and auramine tests in diagnosis of TB lymphadenitis. The incidence of mycobacterial lymphadenitis has increased along with the increase in the incidence of mycobacterial infection worldwide and the highest burden of the disease is found in Asia and Africa. Peripheral lymphadenitis is seen in nearly $35 \%$ of extra-pulmonary tuberculosis which constituted about 15-20\% of all cases of TB. Cervical lymph nodes are the most common site of involvement and reported in 60$90 \%$ of patients with or without involvement of other lymphoid tissue.[9]

\section{METHODS}

This study was a retrospective-prospective study that was carried out in all people suffering from TB lymphadenitis attended Kigali University Teaching Hospital (CHUK), in Rwanda from January 2014 to October 2016. CHUK is located in Kigali Town, Nyarugenge District. To have enlarged lymph node was inclusion criterion and all 137 patients attending CHUK and suffering from lymph node enlargement were enrolled in this study.

\section{Sample collection and preparation}

The samples were collected by doctors assisted by laboratory technicians. The fine needle aspiration technique uses a needle gauge of 23-25 G that is inserted into the lymph node and a certain amount of material is withdrawn. The amount withdrawn was directly put on labelled slides, and the thin smear was dried.

\section{Diff-Quick stain}

In this study the Diff-Quick stain was used. With this Diff-Quick stain nuclear and nucleolar features were less preserved. Cytoplasmic features were better preserved. Solutions required: Fixative (Fast green in methanol), Stain solution 1 (Eosin G in phosphate buffer) and Stain solution 2 (Thiazine dye in phosphate buffer). The following steps were taken in consideration.

To allow air-dry the smear, after drying of smear the four reagents were used as follow: Fix in "DiffQuick" Fixative (or methanol) for 30 secs/drain, Stain with "Diff-Quick" solution II (methylene blue solution) for $30 \mathrm{secs} /$ drain, this was used to stain the nucleus in blue color, Counter stain (optional) with "Diff-Quick" solution I (eosin solution) for 30 secs/drain this used to stain cytoplasm in red or pink color. Rinse in tap water to remove excess stain, rapidly dehydrate in absolute alcohol, Clear and mount. After staining we allowed the slides to dry in air, then the next step is microscopic examination for cytology. In this study the specimens were aspirates or lymph node fluid. After the staining the white blood cells took different color. The platelets appeared violet to purple, neutrophils and nucleus appeared dark blue, cytoplasm appeared pink or red, eosinophils appeared blue sky, granules appeared red to orange, and monocytes appeared violet.

\section{Auramine 0 staining}

\section{Principle of auramine staining}

Acid-fast mycobacteria resist decolorization by acid-alcohol after primary staining due to the high lipid (mycolic acid) content in their cell walls. The identification of mycobacteria with auramine $\mathrm{O}$ is due to the affinity of the mycolic acid in the cell walls for the fluorochromes. The dye will bind to the mycobacteria, which appear as bright yellow, luminous rods against a dark background. The potassium permanganate helps prevent nonspecific fluorescence. All acid-fast organisms will be stained by auramine $\mathrm{O}$, including some parasites. The fluorochromes stains are recommended for specimen examination because of their increased sensitivity and speed.

For auramine $\mathrm{O}$ staining we took in consideration the following procedure: to place the slides, smear upwards, on the staining rack over a sink, about 1 $\mathrm{cm}$ apart. Place a new filter paper in a small funnel, keep it over the first slide and fill it up with auramine staining solution $0.1 \%$. Let the solution filter through the paper, covering each slide completely. Do not heat. Leave for 20 minutes. Using forceps, tilt each slide to drain off the stain solution. Rinse the slides well with distilled water or clean tap water from a beaker (not directly from the tap). Pour the acid solution over the smears, covering them completely, and allow to act for 3 minutes. Using forceps, tilt each slide to drain off the acid-alcohol solution $0.5 \%$. Gently rinse each 
slide again with distilled water or clean tap water from a beaker (not directly from the tap). Flood smears with potassium permanganate $\left(\mathrm{KMnO}_{4}\right)$ $0.5 \%$ or blue ink solution $10 \%$ for 1 minute. Time is critical because counterstaining for longer may quench the AFB fluorescence. Using forceps, tilt each slide to drain off the counter stain solution. Gently rinse each slide again with distilled water or clean tap water from a beaker (not directly from the tap). Using forceps, take each slide from the rack and let the water drain off. Stand the slide on edge on the drying rack and allow to air-dry.

\section{Microscopic examination}

During this step the slides were examined under fluorescent microscope by $40 \times$ objective.

\section{Data analysis}

Samples were tested using all steps of Diff-Quick stain and auramine $\mathrm{O}$ staining. The data collected were analyzed using Microsoft Excel and categorical variable were presented as frequency, percentage $(\%)$ and tables.

\section{Ethical considerations}

This study was carried out at CHUK after approval by ethical committee of CHUK. The names of the patients were not recorded for the ethical reason and it was assured that all personal information has been kept confidentially and used only for study purposes.

\section{RESULTS}

The distribution of lymphadenitis is presented in the table 1.

Table 1: The distribution (number of cases) of lymphadenitis in all suspicious patients for TL.

\begin{tabular}{|l|l|l|l|l|}
\hline Types of lymphadenitis & Male & Female & Total & \% \\
\hline TB lymphadenitis & 32 & 26 & 58 & $42.33 \%$ \\
\hline Necrotizing lymphadenitis & 15 & 12 & 27 & $19.71 \%$ \\
\hline Acute lymphadenitis & 0 & 3 & 3 & $2.19 \%$ \\
\hline Chronic lymphadenitis & 2 & 0 & 2 & $1.42 \%$ \\
\hline Granulomatous lymphadenitis & 10 & 8 & 18 & $13.14 \%$ \\
\hline Reactive lymphadenitis & 20 & 9 & 29 & $21.19 \%$ \\
\hline Total & 79 & 58 & 137 & 100 \\
\hline
\end{tabular}

The total population was 137 suspicious patients for TB lymphadenitis. Some of them were not suffering from TL based on the result of auramine test which is confirmatory test of TB lymphadenitis but they had the same features after Diff-Quick staining and under cytopathology examination. 58 $(42.33 \%)$ cases had TB lymphadenitis.

Table 2: The distribution of auramine and FNA tests in all patients

\begin{tabular}{|l|l|l|l|}
\hline Types/cytological features of TL & FNA test & Auramine test & Total \\
\hline TB Lymphadenitis & 0 & 58 & $42.33 \%$ \\
\hline Necrotizing lymphadenitis & $19.71 \%$ & 0 & $19.71 \%$ \\
\hline Chronic lymphadenitis & $1.42 \%$ & 0 & $1.42 \%$ \\
\hline Acute lymphadenitis & $2.19 \%$ & 0 & $2.19 \%$ \\
\hline Granulomatous lymphadenitis & $13.14 \%$ & 0 & $13.14 \%$ \\
\hline Reactive lymphadenitis & $21.17 \%$ & 0 & $21.17 \%$ \\
\hline Total in $\%$ & $57.67 \%$ & $42.33 \%$ & $100 \%$ \\
\hline
\end{tabular}


Table 2. All patients were 137. In the total patients 58 (42.33\%) had TB lymphadenitis but 79 (57.67) with negative results for auramine test which is confirmatory test for TB lymphadenitis.

Table 3: Distribution of TB lymphadenitis according to gender

\begin{tabular}{|c|c|c|c|}
\hline Type Sex & Male & Female & Total \\
\hline TB lymphadenitis & 32 & 26 & 58 \\
\hline Percentage & $55.17 \%$ & $44.83 \%$ & $100 \%$ \\
\hline
\end{tabular}

Thirty-two (55.17\%) cases were men; the study shows that men are more affected by the TB lymphadenitis

Table 4. The distribution of TB lymphadenitis according to sex and age

\begin{tabular}{|l|l|l|l|l|l|}
\hline Age-years & Female & Male & Total & $\%$ female & $\%$ male \\
\hline $1-15$ & 3 & 8 & 11 & 11.53 & 25 \\
\hline $16-30$ & 8 & 11 & 19 & 30.76 & 34.37 \\
\hline $31-45$ & 12 & 12 & 24 & 46.15 & 37.5 \\
\hline $46-60$ & 3 & 1 & 4 & 11.53 & 3.12 \\
\hline Total & 26 & 32 & 58 & $100 \%$ & $100 \%$ \\
\hline
\end{tabular}

The male and female in the range of 31-45 years were more affected.

\section{DISCUSSION}

The present study focused on the diagnosis of TB lymphadenitis by using fine needle aspiration and auramine $\mathrm{O}$ staining. Fine needle cytology is an easy, reliable procedure for the diagnosis of tubercular lymphadenitis in palpable superficial lymph nodes, and it is ideally suited for use in resource-limited regions, especially in developing countries where TB is a major cause of morbidity and mortality.[16]

In this study 58 (42.33\%) cases were auramine positive for TB lymphadenitis and other cytology discovered $79(57.67 \%)$ cases of suspicious were non TB lymphadenitis.

In this study the observation showed that cytology and auramine $O$ stain for TB lymphadenitis were different in effectiveness. The results obtained in this study show that auramine $\mathrm{O}$ stain conformed TB lymphadenitis diagnosis in comparison with the morphological description of fine needle aspiration, where the auramine was $42.33 \%$ and the confirmatory capacity of fine needle aspiration was $57.67 \%$. The cause of this confirmatory capacity to auramine $\mathrm{O}$ may be the presence of mycolic acid found in cell wall of $M$. tuberculosis [1]. In the previous studies on FNA smears of lymph nodes, autofluorescence was found to be more sensitive than ZN staining,[17] but as compared to cytodiagnosis, it was less sensitive.[18]

In the present study, Males were more affected than females based on the findings where males and females represented $55.17 \%$ and $44.83 \%$ respectively. The TB lymphadenitis was one form of extra-pulmonary tuberculosis. The male and female within the 31-45 years old were more affected where male constituted 12 of 32 (37.5\%) and female 12 of $26(46.15 \%)$ in the positive cases. Although TL was previously considered as a disease of childhood, TB lymphadenitis had a peak age of onset of 20 to 40 years old, and in the United States it is most common in women and immigrants. [8] 
Comparison of the FNA technique to the auramine staining technique shows that these were complementary techniques. Only the presence of acid fast bacilli (AFBs) confirms TB lymphadenitis, however, features observed on the fine needle aspirates and cytology that are associated with tuberculosis were helpful to diagnose tuberculosis. Among TB lymphadenitis cases, $77 \%$ of males and $75 \%$ of females were positive.[19]

The fine needle aspirates could improve the diagnosis of TB lymphadenitis and provide valuable information for appropriate treatment. In similar study fine-needle aspiration was performed on one or two enlarged lymph nodes from each child. Auramine O staining was positive for 56/131 $(42.7 \%)$ cases.[7]

\section{REFERENCES}

1. Knechel NA. Tuberculosis: pathophysiology, clinical features, and diagnosis. Critical care nurse. 2009: 2 (29), 34-43.

2. Kandola P, Meena LS. Extra-pulmonary tuberculosis: Overview, manifestations, diagnostic and treatment techniques. Adv Mater Rev. 2014; 1 (1), 13-19.

3. Mukewar S, Ravi R, Prasad A, Dua SK. Colon tuberculosis: endoscopic features and prospective endoscopic follow-up after anti-tuberculosis treatment. Clin Transl Gastroenterol. 2012;3:e24. [PMC free article] [PubMed] [Google Scholar]

4. Akhan O, Pringot J. Imaging of abdominal tuberculosis. Eur Radiol. 2002; 12:312323. [PubMed] [Google Scholar]

5. Uma D, Vasudevan R, Kaushal K P, Saroj KS, and Arun KS. Abdominal tuberculosis of the gastrointestinal tract. World $J$ Gastroenterol. 2014 Oct 28; 20(40): 1483114840. doi: 10.3748/wjg.v20.i40.14831

6. World Health Organization. Global tuberculosis report 2013. Geneva: WHO. 23 Oct; 2013. Available from: http://apps.who.int/iris/bitstream/ 10665 /91355/1/9789241564656_eng.pdf.

[Google Scholar]

\section{CONCLUSION}

The fine needle aspiration and cytology (FNAC) and auramine tests were useful tests to diagnose the TB lymphadenitis. These techniques were less expensive, quick, safe and effective. The auramine test conforms the diagnosis of TB lymphadenitis. Further study with large sample is needed to emphasize on sensitivity and specificity and to assess the performance of FNA against smear microscopy using either Auramine or Fluorescence Microscopy in the diagnosis of TB lymphadenitis
7. Fanny ML, Beyam N, Gody JC, Zandanga G, Yango F., Manirakiza A., \& Bobossi G. Fine-needle aspiration for diagnosis of tuberculous lymphadenitis in children in Bangui, Central African Republic. BMC pediatrics. 2012; 1 (12), 1.

8. Golden MP, Holenarasipur RV. Extrapulmonary Tuberculosis an overview. Am. Fam. Physician. 2005;1:72 (9): 1761 1968.

9. Nwagbara VI, Asuquo ME., Akpan S, Nwachukwu IE, Nnoli, M, \& Ugbem T. Tuberculous Axillary Lymphadenopathy: A Case Report. Journal of Tropical Diseases. 2013; 2 (30), 87-89.

10. Roozendaal R, Mebius RE., \& Kraal G. The conduit system of the lymph node. International immunology. 2008; 12(20), 1483-1487.

11. Gadre DV, Singh UR, Saxena K, Bhatia A, Talwar V. Diagnosis of tubercular cervical lymphadenitis by FNAC, microscopy and culture. Indian J. Turberc. 1991; 38:25 -8

12. Gupta, PR. Difficulties in managing lymph node tuberculosis. Lung India 2004; 4 (21), 50 .

13. Gatabazi JB, Mukabayire O, Bigirimana V, Ngendahayo L, \& Uwimana I. Fine Needle Aspirate and Cytology (FNAC) as Useful Tool in the Diagnosis of Suspected 
Tuberculous Lymphadenitis in Rwanda,. Mycobacteriology Dis. 2016; 200 (6), 1611068.

14. Buley ID. Fine needle aspiration of lymph nodes. Clinical Patholgy. 1998; 2 (8), 881885 .

15. Dagar V, Heda S, Barsagade A, Mahore S, Ambhore N, Karyakarte $\mathrm{R}$ and Khan $\mathrm{S}$. Comparision of ZN Staining and Fluorescent Microscopy in Detection of Acid Fast Bacilli in Fine Needle Aspiration Smears. IOSR Journal of Dental and Medical Sciences. 2016;15 (8), 79-84.

16. . Wright CA, Hesseling AC, Bamford C, Burgess SM, Warren R, Marais BJ. Fineneedle aspiration biopsy: a first-line diagnostic procedure in paediatric tuberculosis suspects with peripheral lymphadenopathy? International Journal of Tuberculosis and Lung Disease. 2009;13(11):1373-1379. [PubMed] [Google Scholar]
17. Wright $C A$, van der Burg $M$, Geiger $D$, Noordzij JG, Burgess SM, Marais BJ. Diagnosing mycobacterial lymphadenitis in children using fine needle aspiration biopsy: cytomorphology, ZN staining and autofluorescence-making more of less. Diagnostic Cytopathology. 2008;36(4):245251. [PubMed] [Google Scholar]

18. Wright CA, van $\mathrm{Zyl} \mathrm{Y}$, Burgess SM, Blumberg L, Leiman G. Mycobacterial autofluorescence in papanicolaou-stained lymph node aspirates: a glimmer in the dark? Diagnostic Cytopathology. 2004;30(4):257-260. [PubMed] [Google Scholar]

19. Paliwal N, Thakur S, Mullick S \& Gupta K. (2011). FNAC in Tuberculous Lymphadenitis Experience from a teritiary level referral centre. Tuberculosis lymphadenitis. 2011; 3 (53), 67-71. 